\title{
College Health Students’ Knowledge and Perceptions of Exercise Benefits and Dysfunctional Exercise
}

\author{
Ying $\mathrm{Li}^{1}$, Linda A. Keeler ${ }^{2}$, and Megan Julia Jetter ${ }^{3}$ \\ ${ }^{1}$ Community Health at Western Washington University \\ ${ }^{2}$ Sport and Exercise Psychology at Western Washington University \\ ${ }^{3}$ Wilderness Emergency Medical Technician with Olympic Ambulance
}

\begin{abstract}
Background and Purpose: A high percentage of people fail to meet the federal Physical Activity Guidelines while some adults develop dysfunctional exercise. The applications of Self-Determination Theory (SDT) in the field of physical activity suggest that how people are motivated to exercise can lead to different outcomes, such as dysfunctional exercise. Adequate training for health promotion professionals can ensure their competency in successfully promoting healthy exercise. This study assessed the knowledge and perceptions regarding exercise benefits and dysfunctional exercise among Kinesiology and Community Health students - the future health professionals. Methods: A crosssectional study was conducted in a convenience sample of 183 participants from a public university in the northwest region. Results: Both Kinesiology and Community Health majors demonstrated a low level of knowledge regarding dysfunctional exercise, and were inclined to cite controlled rather than autonomous benefits for exercise engagement. Conclusion: Program curriculum changes, such as the coverage of dysfunctional exercise and Self-Determination Theory, should be considered to address the issue.
\end{abstract}

(C) 2016 Californian Journal of Health Promotion. All rights reserved.

Keywords: dysfunctional exercise, exercise dependence, kinesiology major, community health major, professional preparation

\section{Introduction}

The value that a healthy exercise regime provides in disease prevention and health promotion has been clearly documented. However, a relatively new phenomenonexercise dependence/dysfunctional exercise-is recognized as problematic and unhealthy (Reel, 2012). The underlying reasons for exercise dependence are complicated; Reel (2012) commented that exercise promotion messages that are inappropriately conveyed by health promotion professionals could be one contributing factor. Community Health students and Kinesiology students are future workforce members in the areas of health education and promotion. To ensure students' competency in promoting physical activity, it is important to know if Community Health and Kinesiology students' academic training is adequately preparing them to do so. The present study conducted a paper-and-pencil survey among college health students to explore their knowledge and perception of dysfunctional exercise and exercise benefits.

\section{Dysfunctional Exercise/Exercise Dependence}

Regular exercise and physical activity are associated with a range of physical and mental health benefits that contribute to disease prevention and a high quality of life as indicated in the federal Physical Activity Guidelines (USDHHS, 2008). However, it can be detrimental to health when individuals develop maladaptive behaviors. If someone exercises excessively or with unhealthy motivations, this may result in both psychological and physical issues: anxiety, depression, musculoskeletal injuries, exercise-related reproductive dysfunctions, sleep disorders, etc. (Delimaris, 2014; Shroff et al., 2006). Many terms have been used to describe these aforementioned phenomena: dysfunctional exercise, obsessive exercise, obligatory exercise, exercise dependence, or exercise addiction (Adkins \& Keel, 2005; Cook et al., 2013; Hausenblas \& 
Symons Downs, 2002; Reel, 2012). For the present study, the definition and criteria of exercise dependence from Reel and Voelker (2013) was adopted to define maladaptive exercise patterns that can cause physical and psychological harm. Further, exercise dependence is used interchangeably in this manuscript with dysfunctional exercise to expand the scope to include pre-diagnosis problematic exercise.

Depending on the selection of study population and measurement instrument, the prevalence rate of dysfunctional exercise varies from approximately $3 \%$ of the general population, to $6.9 \%$ in sport science undergraduate students, to up to $65 \%$ of runners (Hausenblas \& Symons Downs, 2002; Reel \& Voelker, 2013; Szabo \& Griffiths, 2007). Exercise dependence can either exist alone (primary exercise dependence) or occur alongside other disorders, such as eating disorders (secondary exercise dependence) (Hausenblas \& Symons Downs, 2002). Though secondary exercise dependence tends to be more complicated than primary exercise dependence, both types share the following features (Hausenblas \& Symons Downs, 2002; Reel \& Voelker, 2013): (1) tolerance: the need to increase the amount of exercise to obtain the desired effect; (2) withdrawal: the experience of unpleasant symptoms when exercise stops, or the experience of using exercise as a coping strategy to prevent withdrawal symptoms; (3) intention effects: the tendency to exercise more than originally planned; (4) loss of control: the inability to reduce or stop exercise despite the desire to do so; (5) time: a disproportional amount of time spent in exercise; (6) reductions in other activities; and (7) continuity: the failure to stop exercise practice even when indicated, as with the appearance of recurring physical or psychological harms. Similar to the diagnosis of eating disorders not otherwise specified, there can be situations where someone is exercising in a manner that causes physical and psychological harm and yet they may not meet the criteria outlined above for exercise dependence. In that respect, the term dysfunctional exercise will be used to indicate the broader group of those with unhealthy exercise habits. Overall, exercise becomes dysfunctional, or an addiction, not just based on intention and the amount of physical activity one achieves, but also on why the person is exercising (Reel \& Voelker, 2013).

\section{Self-Determination Theory (SDT) and Dysfunctional Exercise}

To understand the underlying reasons for the phenomenon of dysfunctional exercise, the factor of motivation has been explored extensively. Exercise motivation is often discussed through the application of SelfDetermination Theory (SDT) (Fortier, Duda, Guerin, \& Teixeira, 2012; Teixeira, Carraca, Markland, Silva, \& Ryan, 2012; Wilson, Mack, \& Grattan, 2008). According to the SDT of motivation, people have three basic psychological needs-autonomy, competence, and relatedness-which together foster and nurture a person's psychological growth and wellbeing. Satisfaction of these needs results in improved wellbeing and provides the basis for the development of motivation for behaviors. According to SDT, people can have both intrinsic (inherent satisfaction, joy) or extrinsic (satisfying internalized values, goals, or pressures, or complying with external forces) motivation for behaviors. Specific motivation types are categorized as either autonomous (intrinsic, integrated, identified) or controlled (introjected, external). The type of motivation one has toward an activity influences the degree to which the three basic needs are fulfilled. When the motivation to exercise is influenced primarily by autonomous types (even more so with intrinsic motivation specifically), the basic needs are more likely to be satisfied, thereby leading to a higher success rate of initiating and sustaining exercise behavior (Teixeira et al., 2012). When people approach activities with controlled motivation, their psychological needs are often thwarted, which potentially leads to dissatisfaction and dropout.

The application of SDT in the field of exercise and physical activity suggests that people are more likely to exercise regularly or adhere to long-term exercise regimens if they feel that exercise meets their internal desire to have fun, aligns with their personal values, or results in a sense of accomplishment. Conversely, sedentary individuals are either not motivated to be 
physically active or, perhaps more likely, are motivated by controlled motivators (e.g., appearance, weight loss, scholarships, win/loss record) and have not been able to sustain exercise programs (Teixeira et al., 2012). Furthermore, some exercisers who maintain controlled motivations appear to be at risk for a pattern of dysfunctional exercise (Pritchard \& Beaver, 2012), as they are using exercise as a "means to an end" (i.e., body tone, attractiveness).

Regular exercise can lead to many physical health benefits. Therefore, physical activity promotional messages often cite those types of benefits, such as weight loss, for motivational purposes (Reel, 2012). However, health professionals can accidently contribute to the development of dysfunctional exercise among the public when exercise is promoted as a way to lose or control weight (controlled motivation). The connection between controlled motivation and dysfunctional exercise highlights the need to examine the physical activity promotional messages provided by health professionals and explore the potential deleterious effects that may lead to undesirable outcomes.

\section{Academic Training for Health Promotion Professionals}

There is a growing body of literature on the knowledge of training for health promotion professionals (Brawley, Gierc, \& Locke, 2013; Davidson, 2008). A panel of nationally recognized health education leaders identified "communication" as one of the nine areas of competency that health educators need but often lack (Allegrante, Moon, Auld, \& Gebbie, 2001). Brawley and colleagues (2013) recommended incorporating cognitive behavioral strategies (such as theory-based strategies and/or selfefficacy enhancement) into academic curriculum to ensure Kinesiology students' success in promoting physical activities. Given these recommendations, there are a limited number of examinations of health promotion professionals' training in this area. It is important to conduct research into health professionals' knowledge and perception regarding dysfunctional exercise and exercise benefits, as those professionals' promotional messages are likely to reflect their own knowledge and perception. One meaningful way to potentially accomplish this is to examine future health professionals' academic training to see if they are being appropriately prepared to deliver physical activity promotional efforts that are consistent with healthy, sustained, and beneficial exercise rather than with dysfunctional exercise or dropout. The theoretical model of SDT can be used as a marker to discern these consistencies.

The purpose of this study is to assess Kinesiology and Community Health students' knowledge and perceptions regarding dysfunctional exercise and exercise benefits. Specifically, 1) What is the current health college students' knowledge and perception of dysfunctional exercise and exercise benefits; and 2) What differences exist (if any) between Kinesiology and Community Health majors on knowledge and perception regarding dysfunctional exercise and exercise benefits?

\section{Methods}

\section{Participants}

Undergraduates in Kinesiology and Community Health programs from upper division classes, as well as undergraduates from an Introduction to Communication Sciences and Disorders course-were approached to participate in the current study. A total of 188 respondents completed the survey. Five completed surveys were not used due to either incomplete data or to pre-major status in those participants' academic programs. The final sample included 183 participants with Kinesiology majors ( $\mathrm{n}=71$, $62 \%$ female), Community Health majors ( $\mathrm{n}=$ 42, 92.9\% female), other majors such as Communication Science ( $\mathrm{n}=42,81 \%$ female), or undeclared majors ( $\mathrm{n}=28,92.9 \%$ female). The mean age of participating students was 21.1 years $(\mathrm{SD}=2.38)$, with the majority of students in the senior class standing (50.8\%; $17.5 \%$ Junior, 13.7\% Sophomore, 18\% Freshman) and identifying as White - not Hispanic (82.5\%; 8.7\% Asian or Pacific Islander, 4.4\% Hispanic or Latino/Latina, $1.6 \%$ American Indian or Alaskan Native, 1.6\% Other, and .5\% Black not Hispanic). 


\section{Instruments}

Given the absence of a validated scale that measures knowledge and perceptions in the area of exercise dependence and promotional messages, single item questions were designed by the first two authors for this study specifically. The final version of the selfreport, paper and pencil questionnaire included a total of 15 questions with a variety of formats (i.e., some open-ended, some Likert scale based; please contact lead author for copy). Each item was analyzed separately. Given that the survey was not intended to measure a single concept and not all questions in the survey packet were related, interitem reliability coefficients were not calculated. To determine participants' knowledge regarding dysfunctional exercise, participants were asked to define the term. In addition, participants' familiarity with dysfunctional exercise terminology was measured by requesting that they circle the terms they had heard of. The participants' perception and knowledge of exercise benefits were measured using the following question: "Of all the possible benefits that may be experienced from physical activity/exercise, which would you say are the three most important benefits to share with others?"

\section{Procedures}

Human Subject approval from Institutional Review Board and informed consent by participants were obtained prior to data collection. Course instructors were approached for approval to collect data in classes comprising a large number of either Kinesiology, Community Health, or other majors. Survey data were collected at either the beginning or the end of classes for those students who consented to participate.

\section{Data Analysis}

The research design was a cross-sectional study using a convenience sample to assess current students' knowledge and perception regarding exercise benefits and dysfunctional exercise. Responses were also examined for possible differences between different health-related majors that would cover curriculum on exercise (i.e., Community Health and Kinesiology). For the purpose of analyses, undeclared and other major groups were combined into a general comparison group. Open-ended questionnaire answers were coded by two researchers and compared for agreement before analysis. Participants' definition of dysfunctional exercise was coded for accuracy according to six criteria consistent across literature (Hausenblas \& Symons Downs, 2002; Reel \& Voelker, 2013): exercises in excess, causes issues in other life areas, will persist despite problems (i.e., work, injury), withdrawal symptoms within 24-36 hours, increased tolerance, and extrinsic motivation. For the purposes of this study, two criteria for exercise dependence were considered to be included in students' definition if the following occurred: first, only mentions of controlled motivation were considered relevant in place of the extrinsic motivation criterion, as this is consistent with the current view of SDT; and second, any mention of lack of functioning without exercise was considered to be withdrawal symptoms. A between-groups Analyses of Variances (ANOVA) was used to explore any differences between groups on the number of symptoms reported. Perceived important exercise benefits to be communicated with others were coded into either autonomous or controlled motivation types according to SDT (Ryan \& Deci, 2000), and respondents were given a score of 1 to 3 depending on the number of answers that were considered autonomous. Answers such as enjoyment, stimulation, and mood enhancement were coded as autonomous motivation, while answers suggesting the use of exercise as a means to an end (e.g., cardiovascular health, weight loss) were coded as controlled motivation. A between-groups ANOVA was computed based on the differences in the number of autonomous benefits reported; given that the absence of autonomous motives would indicate the presence of controlled motives, only one ANOVA was conducted. Chisquare analyses were used to compare categorical differences on proportions of answers of prevalence; frequencies were reported when expected cell counts did not meet the minimum count of five. 


\section{Results}

\section{Exercise Dependence Terminology}

Students were asked to recognize and circle any of the 11 dysfunctional exercise terms that were presented. Only seven of the terms were recognized by the majority of students, with anorexia nervosa $(94.7 \%)$, bulimia nervosa (94.2\%), burnout (89.4\%), and excessive exercise $(84.7 \%)$ being the most recognized. Other terms included compulsive exercise (63.5\%), overtraining syndrome (54\%), and muscle dysmorphic disorder (50.3\%). Less than half of the students recognized the following terms: exercise dependence (41.3\%), dysfunctional exercise (29.1\%), orthopraxis $(22.2 \%)$, and negative exercise addiction $(21.7 \%)$. The top four recognized terms were consistent across majors. A higher percentage of Kinesiology students appeared to recognize the terms dysfunctional exercise, exercise dependence, and negative exercise addiction (47.9\%, 46.5\%, and 31\%, respectively), compared to Community Health (33.3\%, 11.9\%, and $11.9 \%)$ and other majors $(38.1 \%, 21.4 \%$, and $19 \%)$. Community Health majors

recognized compulsive exercise (76.2\%) more often than Kinesiology majors (64.8\%) and other majors (54.8\%). All majors appeared to recognize the term excessive exercise at similar rates (Kinesiology $85.9 \%$, Community Health $85.7 \%$, other majors $83.3 \%$ ).

\section{Definition}

Students were asked to define dysfunctional exercise. Participants were not able to accurately identify all six criteria (Hausenblas \& Symons Down, 2002) of dysfunctional exercise. There were several students who did not accurately identify any criterion (Kinesiology 42.3\%, Community Health $33.3 \%$, other $34.3 \%$ ). One Kinesiology student accurately recalled three symptoms; the remaining students only included either one or two symptoms in their definitions. An ANOVA did not reveal differences between numbers of symptoms recalled across majors (see Table 1). Interestingly, $7.4 \%$ of the participants incorrectly believed dysfunctional exercise to be defined as exercise to the point of injury.

\section{Perceived Prevalence and Diagnosis}

Students were asked if they knew one or more persons whom they suspected as having dysfunctional exercise. A Chi-square test indicated no significant association between major and numbers of persons suspected to have dysfunctional exercise; but the relationship did approach a moderate effect size (Pallant, 2010; see Table 2. Approximately one third of each major responded that they knew at least one or more persons with dysfunctional exercise (Community Health 35.7\%, Kinesiology 37.1\%

Table 1.

Means and Standard Deviations of Dysfunctional Exercise (DE) and Prioritized Autonomously Motivated Benefits (AMB), across Student Majors

\begin{tabular}{lcccccccc}
\hline & $\begin{array}{c}\text { Community } \\
\text { Health } \\
(\mathrm{n}=42)\end{array}$ & \multicolumn{1}{c}{$\begin{array}{c}\text { Kinesiology } \\
(\mathrm{n}=71)\end{array}$} & & $\begin{array}{c}\text { Other } \\
(\mathrm{n}=70)\end{array}$ & & & \\
\hline Variable & $\mathrm{M}$ & $\mathrm{DS}$ & $\mathrm{M}$ & $\mathrm{DS}$ & $\mathrm{M}$ & $\mathrm{DS}$ & $\mathrm{p}$ & $\eta^{2}$ \\
\hline $\begin{array}{l}\text { \# of DE symptoms } \\
\text { recalled }\end{array}$ & .83 & .70 & .73 & .74 & .80 & .67 & .733 & .003 \\
$\begin{array}{l}\text { Comfort in } \\
\text { diagnosing DE }\end{array}$ & 2.93 & .89 & 3.31 & .98 & 2.85 & .95 & $.01 *$ & .05 \\
\# of AMBs prioritized & 1.17 & .70 & 1.37 & .84 & 1.38 & .79 & .329 & .012 \\
\hline
\end{tabular}


other 35\%). In an exploration of gender differences, approximately one third of women (35.5\%) and half of men (51.3\%) reported that they did not know someone with dysfunctional exercise, and approximately one third of women (38.3\%) and one out of four men (25\%) thought they knew one or more people with dysfunctional exercise; however, a Chi-square test did not reveal significant gender differences: $\chi_{(3,180)}^{2}=5.31, p=.15$, Cramer's $\mathrm{V}=.17$ (small effect size). Approximately one third of respondents (31.5\%) knew at least one athlete who had dysfunctional exercise, 38.9\% knew

people with dysfunctional exercise who were not an athlete, and 29.6\% were unsure. Approximately one fifth of respondents (21.1\%) knew someone with dysfunctional exercise who did not have a concurrent eating disorder, while $32.1 \%$ knew at least one person who did and $46.8 \%$ were unsure. Almost one out of three students $(31.4 \%)$ was comfortable or very comfortable identifying or diagnosing someone with dysfunctional exercise. There was a significant difference found between majors and comfort in diagnosis: $F_{(2,181)}=4.57, p=.01, \eta^{2}=$ .05 (see Table 1 ).

Education. The top three sources of information about dysfunctional exercise for students from all majors were reading on my own (51.9\%), academic course/lecture (48.6\%), and talking with others (52.5\%). Information was received less from television/movies (28.4\%), special university lectures/seminar series (9.8\%), radio/podcasts (4.4\%), and professional conferences (2.7\%). Only $4.2 \%$ of the respondents were not interested in learning more about dysfunctional exercise versus $63.5 \%$ who were interested and $31.7 \%$ who might be interested. The top methods by which students preferred to be educated about dysfunctional exercise were consistent across majors: traditional lecture $(52.5 \%)$, reading on one's own (44.8\%), and an expert panel (24\%).

\section{Exercise Benefits}

Students were asked to list the three most important exercise benefits to communicate with others, and answers were coded according to which motivation types the benefits corresponded to according to SDT (Ryan \& Deci, 2000). Examples of important exercise benefits reported were coded into either autonomously related (e.g., confidence, feel good, enjoyment/fun) or controlled related (e.g., lose weight, prevent heart disease, health). It should be noted that an answer of "health" can be accurately categorized in either autonomous or controlled motivations depending on the meaning and intention of the word (e.g., being active because living a healthy lifestyle is valued and a part of self-identity would be autonomous motivation, vs. exercising as a means to gain or prevent a health-related concept such as blood sugar or body composition would be controlled

Table 2.

Percentage of Respondents with Knowledge of Someone with Dysfunctional Exercise (DE) and Motive Types of Benefits across Student Majors

\begin{tabular}{lccccc}
\hline & $\begin{array}{c}\text { Community } \\
\text { Health } \\
(\mathrm{n}=42)\end{array}$ & $\begin{array}{c}\text { Kinesiology } \\
(\mathrm{n}=71)\end{array}$ & $\begin{array}{c}\text { Other } \\
(\mathrm{n}=70)\end{array}$ & $p$ & $\begin{array}{c}\text { Cramer's } \\
\mathrm{V}\end{array}$ \\
\hline $\begin{array}{l}\text { Knew at least one person } \\
\text { with DE }\end{array}$ & $35.7 \%$ & $37.1 \%$ & $35 \%$ & .098 & .17 \\
$\begin{array}{l}\text { Prioritized more AM than } \\
\mathrm{CM}^{\mathrm{a}}\end{array}$ & $28.6 \%$ & $42.9 \%$ & $44.9 \%$ & ---- & ---- \\
\hline
\end{tabular}

Note. $\mathrm{AM}=$ autonomous motives; $\mathrm{CM}=$ controlled motives.

${ }^{a}$ Cell count lower than expected prevented use of Chi-square analysis. 
motivation). Due to the probability that educational messages in the Community Health, and, possibly, the Kinesiology majors may promote health in a prevention- and treatmentbased approach, it was assumed that an answer of "health" by this sample would likely denote exercising as a means to an end; thereby, it was coded as a controlled motivation.

The three types of major students did not differ significantly on the mean amount of autonomous motivation types listed: $\mathrm{F}_{(2,178)}=1.12, p=.329$, $\eta_{2}=.012$, with a small effect size (see Table 1 ). It should be noted that all three groups' means were less than two, indicating that all three groups reported more controlled motives than autonomous motives. Upon visual inspection, more Kinesiology and other majors reported more autonomous motives than controlled motives compared to Community Health students (see Table 2), although this accounted for less than half of each group of student majors. The largest sub-group for all majors was one autonomous motivation and two controlled motivations (Community Health 57.1\%, Kinesiology 42.9\%, other 42\%). In an exploration of gender differences, the largest sub-groups of females (50.7\%) stated one autonomous and two controlled benefits, while men $(43.6 \%)$ stated two autonomous and one controlled benefits. The majority of both men and women stated more controlled than autonomous motivations.

\section{Discussion}

This study examined knowledge and perceptions of dysfunctional exercise and exercise benefits among students in health related majors. Our findings support that participants may benefit from receiving lectures or additional training in the field of dysfunctional exercise, as many participants could not recognize the major terms related to dysfunctional exercise or identify the diagnosis criteria. Regarding their abilities in term recognition, participants' capability of identifying diagnosis criteria could be better as $42.3 \%$ of Kinesiology, 33.3\% of Community Health, and $34.3 \%$ of other majors could not identify any criterion. Out of six criteria, most students could identify one or two criteria, and there did not appear to be significant variances in the number of symptoms identified across different majors.

The prevalence of dysfunctional exercise was estimated through participants' observations, with an average of $31.5 \%$ across Kinesiology, Community Health, and other majors knowing at least one or more persons with it, which is within the range of $.3 \%-52 \%$ in the literature (Cook et al., 2013). It is not surprising that the female participants were more likely to know someone with dysfunctional exercise given that eating disorders are more prevalent among women and secondary exercise dependence may be comorbid symptomology - that is, if one assumes that women have more female friends than men. At the same time, readers should be cautious with the result, as the participants demonstrated low competency in defining the term and listing the diagnosis criteria.

It is interesting, but not surprising, that the participants listed talking with others and reading on my own as the top two information sources for dysfunctional exercise, followed by academic course/lecture. There can be misinformation shared by others or through certain reading material, which may explain the reason why Kinesiology and Community Health majors did not perform better than other majors in terminology and diagnosis criteria recognition. This information on the source of knowledge again points to the need to include this topic in the program curriculum for both majors.

Students were asked which top three benefits of exercise were the most important to share with others. The purpose of this question was to identify respondents' beliefs and values about which motivation types they had learned to promote. It was surprising that all participants tended to list more controlled benefits than autonomous ones. According to SDT, people with controlled motivation are less likely to initiate and sustain exercise in comparison with people with autonomous motivation (Ng et al., 2012), which may partially explain the reality that the majority of adults fail to meet the Federal Physical Activity 
Guidelines for both aerobic and musclestrengthening activity (Ward, Schiller, \& Freeman, 2014). In their efforts to promote exercise, health professionals should emphasize benefits that are more in line with autonomous motivation (such as internal joy) rather than benefits that can be perceived as controlled motivation. Contradictorily, both Community Health (28.6\%) and Kinesiology majors (42.9\%) — the presumed future health promotion professionals in the current sample-were less likely to list more autonomous benefits than other majors (44.9\%). This is particularly concerning because controlled motivation is a common occurrence for those who have dysfunctional exercise, so the messages about which motivational benefits are important to promote to the public could be vital in its prevention. It is unclear whether students who already have more controlled motivators for exercise select health-related majors, or whether the curriculum influenced these perceived benefits. Regardless, this discrepancy warrants program curriculum examination and future exploration to shed light on the underlying reasons for this phenomenon. At the same time, it is worth mentioning the gender differences in citing exercise benefits. Females were more likely to list more controlled motives (e.g., improved health, disease prevention) than autonomous ones (e.g., feel good, enjoyment), which may be explained by the media socialization influence of unrealistic body image.

To have an accurate and comprehensive understanding of the results, the readers should be aware of the coding approach used in this study. Health benefits from exercise, which can either be autonomous or controlled depending on how much participants value health and to what degree they internalize this benefit, were mentioned quite often among the participants. For our study, all health benefits were coded as controlled benefits, which may skew the results.
For future research, more specific directions should be included to encourage participants to be more specific as to which health benefit was being referred to and why. This clarification could improve the accuracy of the coding for controlled and autonomous health benefits.

\section{Limitations}

The current study had limitations. First, all information was self-reported and was therefore subject to social desirability response bias; however, the emphasis of anonymity at the beginning of the survey encouraged honest answers. Second, a convenience sample of participants who were predominately senior-year students and delimited to two majors in the same department on the same campus limited the generalizability of the results. Third, the study included newly constructed questions aimed at measuring some knowledge and perceptions, but was not a rigorously tested survey meant to measure the full concept of exercise dysfunction and benefit knowledge.

\section{Implications for Research and Practice}

The major takeaway message from this study is that both Community Health and Kinesiology majors may not be well informed and trained in dysfunctional exercise. To improve this situation, university programs that prepare Community Health and Kinesiology majors should include at least one or two lectures about dysfunctional exercise to discuss the terminology, symptoms, diagnosis criteria, and current research. In addition, it is important to educate both majors about Self-Determination Theory to have a better understanding of how autonomous and controlled motivation may influence people's behaviors and possibly lead to dysfunctional exercise or exercise dropout. More importantly, practical application of SDT should be discussed-specifically, how to phrase a message to increase people's intrinsic and autonomous motivation for physical activity and exercise. 


\section{References}

Adkins, E. C., \& Keel, P. K. (2005). Does “excessive” or “compulsive” best describe exercise as a symptom of bulimia nervosa? International Journal of Eating Disorders, 38(1), 24-29. doi:10.1002/eat.20140

Allegrante, J. P., Moon, R. W., Auld, E., \& Gebbie, K. M. (2001). Continuing education needs of the currently employed public health education workforce. American Journal of Public Health, 91, 1230-1234. doi:10.2105/ajph.91.8.1230

Brawley, L. R., Gierc, M. S. H., \& Locke, S. R. (2013). Powering adherence to physical activity by changing self-regulatory skills and beliefs: Are kinesiologists ready to counsel? Kinesiology Review, 2, 4-16. doi:10.1123/krj.2.1.4

Cook, B., Karr, T., Zunker, C., Mitchell, J., Thompson, R., Sherman, R., ...Wonderlich, S. (2013). Primary and secondary exercise dependence in recreational road race runners. Journal of Sport and Exercise Psychology, 35, 464-469. doi:10.1016/s0161-6420(01)00552-8

Davidson, E. S. (2008). Perceived continuing education needs and job relevance of health education competencies among health education and promotion practitioners in college health settings. Journal of American College Health, 57(2), 197-104. doi:10.3200/jach.57.2.197-210

Delimaris, I. (2014). Potential adverse biological effects of excessive exercise and overtraining among healthy individuals. Acta Medica Martiniana, 14(3), 5-12. doi:10.1515/acm-2015-0001

Fortier, M. S., Duda, J. L., Guerin, E., \& Teixeira, P. J. (2012). Promoting physical activity: Development and testing of self-determination theory-based interventions. International Journal of Behavioral Nutrition and Physical Activity, 9, 1-14. doi:10.1186/1479-5868-9-20

Hausenblas, H. A., \& Symons Downs, D. (2002). Exercise dependence: A systematic review. Psychology of Sport and Exercise, 3, 89-123. doi:10.1016/S1469-0292(00)00015-7

Ng, Y. Y., Ntoumanis, N., Thøgersen-Ntoumani, C., Deci, E., Ryan, R., Duda, J., \& Williams, G. C. (2012). Self-determination theory applied to health contexts: A meta-analysis. Perspectives on Psychological Science, 7(4), 325-340.

Pallant, J. (2010). SPSS survival manual. (4 $4^{\text {th }}$ ed.). New York, NY: Open University Press.

Pritchard, M. E., \& Beaver, J. L. (2012). Do exercise motives predict obligatory exercise? Eating Behaviors, 13(2), 139-141. doi:10.1016/j.eatbeh.2011.11.012

Reel, J. J. (2012). The right 'dose' of activity: Health educators should promote mindful and intuitive exercise. Journal of Community Medicine and Health Education, 2(9), 1-2. doi:10.4172/2161$0711.1000 \mathrm{e} 111$

Reel, J. J., \& Voelker, D. (2013). Exercise to the extreme? Identifying and addressing unhealthy exercise behaviors. Athletic Insight, 15, 301-314.

Ryan, R. M., \& Deci, E. L. (2000). Self-determination theory and the facilitation of intrinsic motivation, social development, and well-being. American Psychologist, 55, 68-78.

Shroff, H., Reba, L., Thornton, L. M., Tozzi, F., Klump, K. L., Berrettini, W. H., \& Bulik, C. (2006). Features associated with excessive exercise in women with eating disorders. International Journal of Eating Disorders, 39(6), 454-461. doi:10.1002/eat.20247

Szabo, A., \& Griffiths, M. D. (2007). Exercise addiction in British sport science students. International Journal of Mental Health and Addiction, 5, 25-28.

Teixeira, P. J., Carraça, E. V., Markland, D. A., Silva, M. N., \& Ryan, R. M. (2012). Exercise, physical activity, and self-determination theory: A systematic review [Electronic version]. Journal of Behavioral Nutrition and Physical Activity, 9, 78. doi:10.1186/1479-5868-9-78

Ward, B. W., Schiller, J. S., \& Freeman, G. (2014). Early release of selected estimates based on data from the January-September 2013 National Health Interview Survey. Atlanta, GA: National Center for Health Statistics. Retrieved from: http://www.cdc.gov/nchs/nhis.htm

Wilson, P. M., Mack, D. E., \& Grattan, K. P. (2008). Understanding motivation for exercise: A selfdetermination theory perspective. Canadian Psychology, 49, 250-256.

United States Department of Health and Human Services (USDHHS). (2008). 2008 Physical Activity Guidelines for Americans. Washington, DC: USDHHS. 


\section{Corresponding Author Information}

Dr. Ying Li

Associate Professor in Community Health

Western Washington University

ying.li@wwu.edu

Bond Hall 103, Department of HDD

Western Washington University

Bellingham, WA, 98225

(360)650-3513 